\title{
XANTATINA INHIBE LA ACTIVACIÓN DE MASTOCITOS INDUCIDA POR NEUROPÉPTIDOS PRO-INFLAMATORIOS
}

\author{
Patricia M Vargas ${ }^{1-2}$, Elia Martino ${ }^{2-3}$, Teresa H Fogal ${ }^{1}$, Carlos E Tonn ${ }^{4}$, Alicia B \\ Penissi $^{1^{*}}$ \\ ${ }^{1}$ Área e Instituto de Histología y Embriología "Dr. Mario H. Burgos" (IHEM-CONICET), Facultad de Ciencias \\ Médicas, Universidad Nacional de Cuyo, Mendoza, Argentina.
${ }^{2}$ Área de Anatomía Normal, Facultad de Ciencias Médicas, Universidad Nacional de Cuyo, Mendoza, Argentina. \\ ${ }^{3}$ Servicio de Neurofisiología, Hospital El Carmen. Mendoza, Argentina. \\ ${ }^{4}$ INTEQUI-CONICET-UNSL, Departamento de Química, Facultad de Química, Bioquímica y Farmacia, \\ Universidad Nacional de San Luis, San Luis, Argentina.
}

\begin{abstract}
RESUMEN
Los mastocitos son células del tejido conectivo que participan en la génesis y modulación de las respuestas inflamatorias. Previamente hemos demostrado que xanthatina (xanthanólido sesquiterpeno aislado de Xanthium cavanillesii Schouw) inhibe la activación de mastocitos inducida por secretagogos experimentales. Sin embargo, se desconoce su efecto sobre la activación de mastocitos inducida por estímulos fisiopatológicos. Estos estímulos incluyen, entre otros, los neuropéptidos pro-inflamatorios sustancia $P$ y neurotensina, responsables de una de las principales vías de inflamación neurogénica. El objetivo del presente trabajo fue estudiar el efecto de xanthatina sobre la activación de mastocitos inducida por sustancia $P$ y neurotensina. Mastocitos peritoneales de rata se incubaron con: 1) PBS (basal); 2) sustancia $P(100 \mu \mathrm{m}) ; 3)$ neurotensina $(50 \mu \mathrm{m}) ; 4)$ xanthatina $(8-320 \mu \mathrm{m})+$ sustancia $P$; 5$)$ xanthatina (8$320 \mu \mathrm{m})+$ neurotensina. Se llevaron a cabo los siguientes estudios: análisis dosis-respuesta de la liberación de serotonina inducida por neuropéptidos proinflamatorios, vitalidad celular, morfología mastocitaria por microscopía óptica y electrónica, análisis de estabilidad de xanthatina por cromatografía en capa fina. Los ensayos de liberación de serotonina y los estudios morfológicos mostraron la efectividad de xanthatina para estabilizar mastocitos. El presente estudio provee la primer evidencia a favor de la hipótesis de que xanthatina inhibe la liberación de serotonina inducida por sustancia $P$ y neurotensina $a$ partir de mastocitos peritoneales. Este sesquiterpeno podría representar una nueva alternativa fármacológica en la regulación de la activación mastocitaria para el tratamiento de las inflamaciones neurogénicas.
\end{abstract}

Palabras clave: xanthanólido, sustancia $P$, neurotensina

\begin{abstract}
Mast cells are connective tissue cells involved in the genesis and modulation of inflammatory responses. We have previously shown that xanthatin (xanthanolide sesquiterpene isolated from Xanthium cavanillesii Schouw) inhibits mast cell activation induced by experimental secretagogues. However, the effect of xanthatin on mast cell activation induced by pathophysiological stimuli remains unknown. These stimuli include, among others, the pro-inflammatory neuropeptide substance $P$ and neurotensin, responsible for one of the main pathways of neurogenic inflammation. The present study was designed to examine the effects of xanthatin on mast cell activation induced by pro-inflammatory peptides, such as substance $P$ and neurotensin. Rat peritoneal mast cells were incubated with: 1) PBS (basal); 2) substance $P(100 \mu \mathrm{m})$; 3) neurotensin $(50 \mu \mathrm{m}) ; 4)$ xanthatin $(8-320 \mu \mathrm{m})+$ substance $P ; 5)$ xanthatin $(8-320$ $\mu \mathrm{m})+$ neurotensin. Concentration-response studies of mast cell serotonin release evoked by pro-inflammatory neuropeptides, evaluation of mast cell viability and morphology by light and electron microscopy, and drug stability analysis by thin layer chromatography were performed. Serotonin release studies, carried out together with morphological studies, showed the effectiveness of xanthatin to stabilize mast cells. The present study provides the first strong evidence in favour of the hypothesis that xanthatin inhibits substance $P$ - and neurotensin-induced serotonin release from peritoneal mast cells. Our findings may provide an insight into the design of novel pharmacological agents which may be used to regulate the mast cell response in neurogenic inflammation.
\end{abstract}

Key words: xanthanolide - substance $P$ - neurotensin

* Correspondencia a: Alicia B. Penissi, Casilla de Correo 56. (5500) Mendoza, Argentina. apenissi@fcm.uncu.edu.ar

Recibido: 26 de diciembre de 2009. Revisado:11 de febrero de 2010. Aceptado:8 de marzo de 2010. 


\section{INTRODUCCIÓN}

Los mastocitos son células especializadas del tejido conectivo que liberan, en respuesta a la activación por estímulos externos, una gran variedad de mediadores biológicamente activos (Kalesnikoff y Galli, 2008; Galli et al, 2008; Rao y Brown, 2008).

Por el hecho de estar especialmente concentrados en tejidos que se encuentran en contacto con el medio externo (piel, tracto respiratorio, tubo digestivo, vías genitourinarias), son los primeros en detectar un daño tisular, lo que provoca la liberación de mediadores responsables de reclutar a los polimorfonucleares e iniciar las respuestas características de una inflamación aguda (Galli et al, 2008; Kubes y Granger, 1996). Los mastocitos pueden inducir de esta manera la activación de células del sistema inmune como granulocitos, monocitos/ macrófagos, células dendríticas, linfocitos $\mathrm{T}$, linfocitos B y linfocitos NK (Galli et al, 2008).

Los mastocitos juegan un papel central en la patogénesis de enfermedades como el asma, rinoconjuntivitis, urticaria, ciertos tipos de cáncer, anafilaxis, alergias alimentarias, mastocitosis sistémica, enfermedad inflamatoria intestinal, artritis reumatoidea, dermatitis atópica, cistitis intersticial, síndrome del colon irritable, migrañas, esclerosis múltiple e inflamación neurogénica (Rao y Brown, 2008; Gurish y Austen, 2001; Schmelz y Petersen, 2001).

Se han identificado dos vías de activación de mastocitos (Kalesnikoff y Galli, 2008; Blank y Rivera, 2004): 1) Vía inmunológica: es dependiente de la agregación de los receptores de alta afinidad para el fragmento Fc de las inmunoglobulinas $E$ (FcerI) y 2) Vía no inmunológica: es independiente de la agregación de los FceRI. Entre los secretagogos básicos de vía no inmunológica destacan los neuropéptidos pro-inflamatorios sustancia $P$ y neurotensina. La estimulación de mastocitos inducida por estos neuropéptidos constituye una de las principales vías de inflamación neurogénica (Rao y Brown, 2008; Richardson y Vasko, 2002; Moriyama et al, 2005).

El proceso de activación mastocitaria culmina con tres respuestas diferentes (Kushnir-Sukhov et al, 2007): a) Exocitosis de los gránulos secretorios que contienen moléculas bioactivas preformadas (degranulación), como la histamina y la serotonina; b) Síntesis "de novo" y secreción de mediadores lipídicos.

En trabajos previos hemos demostrado que xanthatina (sesquiterpeno de núcleo xanthanólido $\alpha, \beta$-insaturado aislado de Xanthium cavanillesii Schouw), previene la formación de lesiones gastrointestinales inducidas por agentes ulcerogénicos y ejerce una potente actividad antiinflamatoria (Giordano et al, 1990; Penissi et al, 1998; Penissi et al, 1999; Penissi et al, 2000; Penissi et al, 2006). Hemos demostrado además que xanthatina inhibe la liberación de histamina y serotonina inducida por secretagogos experimentales como el compuesto 48/80 en mastocitos de roedores (Penissi et al, 2003b; Penissi et al, 2003a; Penissi et al, 2003c; Penissi et al, 2009). Sin embargo, se desconoce el efecto de xanthatina sobre la activación de mastocitos inducida por estímulos fisiopatológicos.

El presente trabajo pretende estudiar la acción de xanthatina sobre la activación de mastocitos inducida por neuropéptidos proinflamatorios. Este sesquiterpeno podría representar una nueva estrategia farmacológica en el tratamiento de patologías inflamatorias vinculadas con la activación de mastocitos, como la inflamación neurogénica.

\section{MATERIAL Y MÉTODO}

\section{Xanthatina}

El aislamiento y purificación de xanthatina se llevó a cabo a partir de la porción aérea de Xanthium cavanillesii Schouw (Favier et al, 2005). Brevemente, las hojas secas de esta planta $(4,8 \mathrm{~kg})$ se extrajeron con acetona, tres veces, a temperatura ambiente durante cinco días. El extracto fue concentrado bajo vacío y el producto resultante $(560 \mathrm{~g})$ fue disuelto en una mezcla de metanol:agua (9:1), filtrado y extraído con n-hexano para remover pigmentos y materiales grasos. La solución hidroalcohólica fue diluída con agua (7:3) y luego extraída con cloroformo. Luego de la evaporación de los solventes, se fraccionó el residuo resultante por cromatografía flash y cromatografía en columna. Este protocolo de purificación rindió xanthatina (3,8 g, $100 \%$ de pureza), que fue identificada por resonancia magnética nuclear de ${ }^{1} \mathrm{H}$ y ${ }^{13} \mathrm{C}$, espectrometría de masa, espectro infrarrojo y análisis de punto de fusión.

\section{Animales}

Se utilizaron ratas machos adultas de la cepa Sprague-Dawley, mantenidas en el bioterio del Instituto de Histología y Embriología (IHEM) bajo estricto control de alimentación, temperatura $\left(22 \pm 1^{\circ} \mathrm{C}\right)$ y períodos de luz/oscuridad (luz de 6:00 a 18:00 y oscuridad de 18:00 a 6:00). Todos los experimentos se llevaron a cabo de acuerdo 
con la Guía para el Cuidado y Uso de Animales de Laboratorio de los Institutos Nacionales de Salud (NIH, USA). Los protocolos utilizados han sido aprobados por el Comité Institucional de Cuidado y Uso de Animales de Experimentación (CICUAL) de la Facultad de Ciencias Médicas de la Universidad Nacional de Cuyo.

Aislamiento y purificación de mastocitos peritoneales

Los animales se sacrificaron con éter etílico bajo una campana de extracción. Inmediatamente después del sacrificio, los mastocitos fueron aislados por lavado peritoneal y luego purificados en un gradiente discontinuo de solución estéril de Percoll (Calderón et al, 1998). La pureza de las células aisladas se evaluó por tinción con azul de toluidina al $1 \%$ en ácido clorhídrico $(\mathrm{HCl}) 0,7 \mathrm{~N}$. Para los diferentes ensayos se utilizaron las preparaciones con una pureza del $95-100 \%$. La viabilidad de los mastocitos luego de la purificación se analizó por exclusión del colorante azul tripán.

\section{Protocolo general}

Los mastocitos peritoneales fueron incubados con: 1) Solución buffer fosfato salino (PBS): grupo basal. Tiempo de incubación: 20 minutos. 2) Sustancia $P$ : se ensayaron diferentes concentraciones de sustancia $\mathrm{P}(1 \mu \mathrm{M}, 50 \mu \mathrm{M}$ y $100 \mu \mathrm{M})$ para establecer la dosis óptima de activación en nuesto sistema experimental. Tiempo de incubación: 10 min con solución PBS y 10 minutos adicionales con sustancia $P$. 3) Neurotensina: se ensayaron diferentes concentraciones de neurotensina $(1 \mu \mathrm{M}, 50 \mu \mathrm{M}$ y 100 $\mu \mathrm{M})$ para establecer la dosis óptima de activación en nuesto sistema experimental. Tiempo de incubación: 10 min con solución PBS y 10 minutos adicionales con neurotensina. 4) Xanthatina+sustancia $\mathrm{P}(100 \mu \mathrm{M})$ : se utilizaron diferentes dosis de xanthatina $(8 \mu \mathrm{M}, 16 \mu \mathrm{M}, 40$ $\mu \mathrm{M}, 80 \mu \mathrm{M}, 160 \mu \mathrm{M}$ y $320 \mu \mathrm{M})$ para construir curvas dosis-respuesta. Tiempo de incubación: 10 min con xanthatina y 10 minutos adicionales con sustancia P. 5) Xanthatina+neurotensina (50 $\mu \mathrm{M})$ : se utilizaron diferentes dosis de xanthatina $(8 \mu \mathrm{M}, 16 \mu \mathrm{M}, 40 \mu \mathrm{M}, 80 \mu \mathrm{M}, 160 \mu \mathrm{M}$ y $320 \mu \mathrm{M})$ para construir curvas dosis-respuesta. Tiempo de incubación: 10 min con xanthatina y 10 minutos adicionales con neurotensina.

La viabilidad de los mastocitos luego de las incubaciones se analizó por exclusión del colorante azul tripán. En las soluciones de incubación se cuantificó serotonina como marcador de degranulación por Cromatografía Líquida de Alta Resolución (HPLC) con detección electroquímica. En las células se estudió la concentración de serotonina remanente (no liberada) luego de las incubaciones, la morfología de los mastocitos por microscopía óptica con azul de toluidina y microscopía electrónica de transmisión. La pureza y estabilidad de xanthatina antes y después de las incubaciones fue evaluada por cromatografía en capa fina (TLC).

\section{Cuantificación de serotonina}

Los niveles de serotonina, tanto en las soluciones de incubación como en las células remanentes, fueron medidos por cromatografía líquida de alta resolución (HPLC) con detección electroquímica de acuerdo con el método de Wagner et al (1982) con algunas modificaciones (Penissi et al, 1998). Los resultados se expresaron como porcentaje de liberación de serotonina.

\section{Microscopía óptica con azul de toluidina}

Las muestras fueron fijadas en una solución de glutaraldehído $5 \%$ y formaldehído $4 \%$ en buffer cacodilato $0,1 \mathrm{M}(\mathrm{pH}=7,2)$. Después de $1 \mathrm{~h}$ en el fijador, las células fueron resuspendidas y alícuotas de las resuspensiones fueron colocadas sobre portaobjetos, coloreadas con azul de toluidina al $1 \%$ en $\mathrm{HCl} 0.7 \mathrm{~N}$, observadas y fotografiadas bajo un microscopio óptico acoplado a una cámara fotográfica digital.

\section{Microscopía electrónica de transmisión}

Las muestras fueron fijadas en solución de glutaraldehído al $2.5 \%$ en PBS $(\mathrm{pH}=7,4)$. Después de $1 \mathrm{~h}$ en el fijador, las células fueron refijadas en $\mathrm{OsO}_{4}$ al $1 \%$ en buffer cacodilato $0.2 \mathrm{M}$ en oscuridad durante $12 \mathrm{~h}$. El material fue deshidratado e incluído en Spurr. Se cortaron secciones ultrafinas $(60 \mathrm{~nm})$ con ultramicrótomo se las coloreó con acetato de uranilo y plomo, se las montó sobre grillas, se las examinó en un microscopio electrónico de transmisión y se tomaron micrografías.

\section{Cromatografía en capa fina (TLC)}

Las soluciones de incubación fueron estudiadas por TLC, antes y después de las incubaciones, para analizar la estabilidad química de la xanthatina. Extractos etílicos de las soluciones de incubación y los standards de xanthatina fueron sembrados en placas para TLC. La cromatografía se desarrolló con acetato de etilo/n-hexano y se reveló con permanganato de potasio. Se calcularon las relaciones de frente (Rf) de las bandas obtenidas.

\section{Tratamiento estadístico}

Las diferencias entre los grupos fueron determinadas utilizando análisis de la varianza de tipo 1 (ANOVA-1) seguido por la prueba de Tukey-Kramer. $\mathrm{P}<0,05$ fue considerado estadísticamente significativo. 


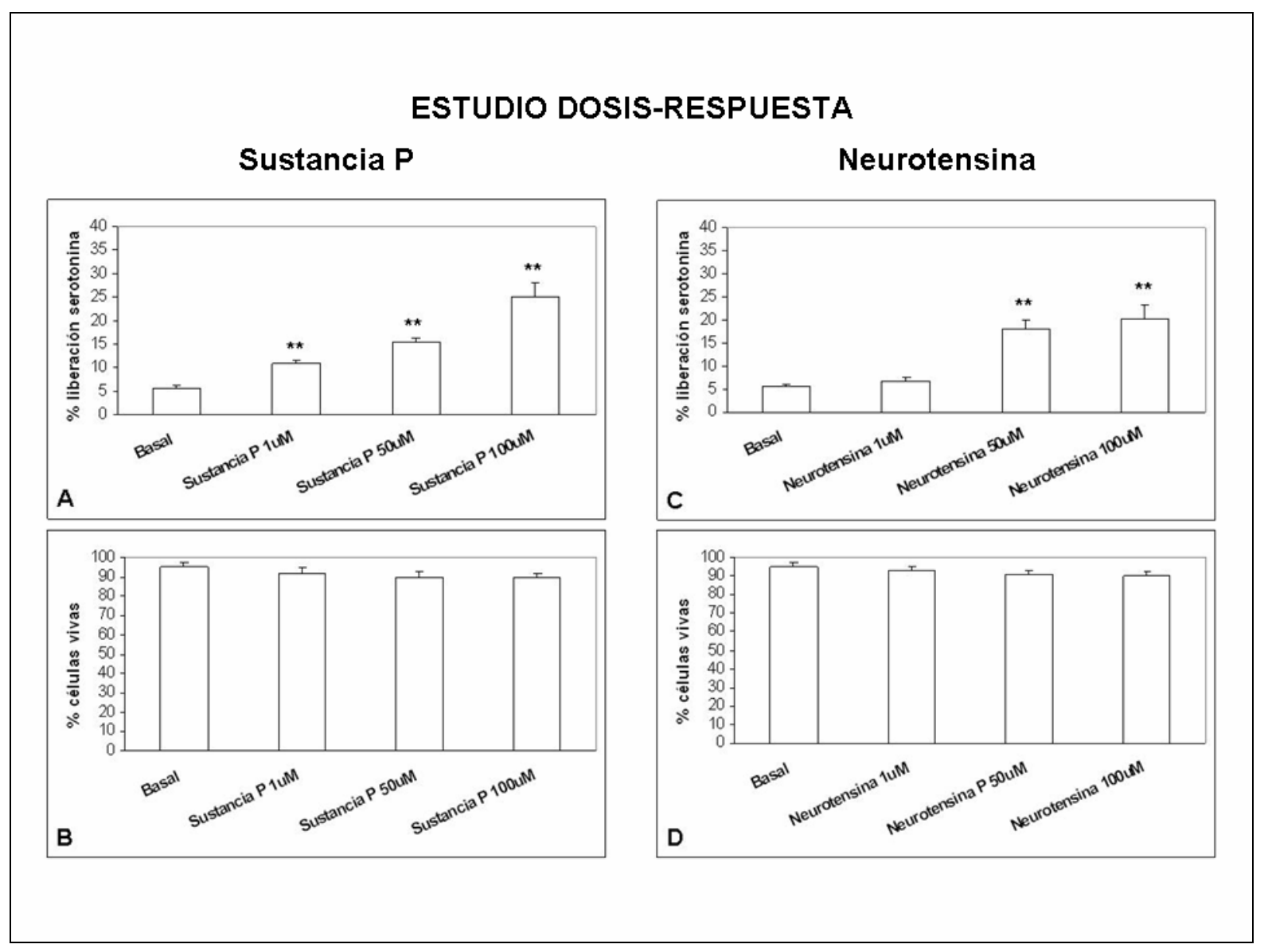

Figura 1. A: Efecto de concentraciones crecientes de sustancia $P$ sobre la liberación de serotonina mastocitaria. B: Efecto de concentraciones crecientes de sustancia P sobre la vitalidad mastocitaria. C: Efecto de concentraciones crecientes de neurotensina sobre la liberación de serotonina mastocitaria. D: Efecto de concentraciones crecientes de neurotensina sobre la vitalidad mastocitaria. Los resultados se expresaron como porcentaje de liberación de serotonina ( $A$ y C) o como porcentaje de células vivas (B y D). Los valores se presentan como media \pm S.E.M. ${ }^{* *} \mathrm{P}<0,01$ versus basal.

\section{RESULTADOS}

\section{Estudios bioquímicos}

La incubación de mastocitos con sustancia $P$ (dosis de $1 \mu \mathrm{M}, 50 \mu \mathrm{M}$ y $100 \mu \mathrm{M}$ ) indujo un incremento significativo $(P<0,01)$ de la liberación de serotonina con respecto al grupo basal (Fig. 1A). Ninguno de los grupos experimentales mostró cambios en la viabilidad celular (Fig. 1B). Teniendo en cuenta estos resultados se eligió la dosis de sustancia $\mathrm{P} 100 \mu \mathrm{M}$ para realizar los ensayos con xanthatina.

La incubación de mastocitos con neurotensina 1 $\mu \mathrm{M}$ no modificó la liberación de serotonina. La incubación de mastocitos con neurotensina 50 $\mu \mathrm{M}$ y $100 \mu \mathrm{M}$ indujo un incremento significativo $(P<0,01)$ de la liberación de serotonina con respecto al grupo basal. La liberación inducida por neurotensina $50 \mu \mathrm{M}$ fue similar a observada frente a la acción de neurotensina $100 \mu \mathrm{M}$ (Fig. 1C). Ninguno de los grupos experimentales mostró cambios en la viabilidad celular (Fig. 1D). Teniendo en cuenta estos resultados se eligió la dosis de neurotensina $50 \mu \mathrm{M}$ para realizar los ensayos con xanthatina.

La incubación de mastocitos con xanthatina (dosis de $8 \mu \mathrm{M}, 16 \mu \mathrm{M}, 40 \mu \mathrm{M}$ y 80) inhibió $(P<0,01)$, en forma dosis-dependiente, la liberación de serotonina inducida por sustancia $P$ $100 \mu \mathrm{M}$ (Fig. 2A), sin modificar la viabilidad celular (Fig. 2B). La incubación de las células con xanthatina $160 \mu \mathrm{M}$ y $320 \mu \mathrm{M}$ aumentó la liberación de serotonina con respecto a la dosis de $80 \mu \mathrm{M}$ (Fig. 2A) y disminuyo significativamente $(P<0,01)$ la vitalidad celular (Fig. 2B). En base a estos resultados, se seleccionó la 


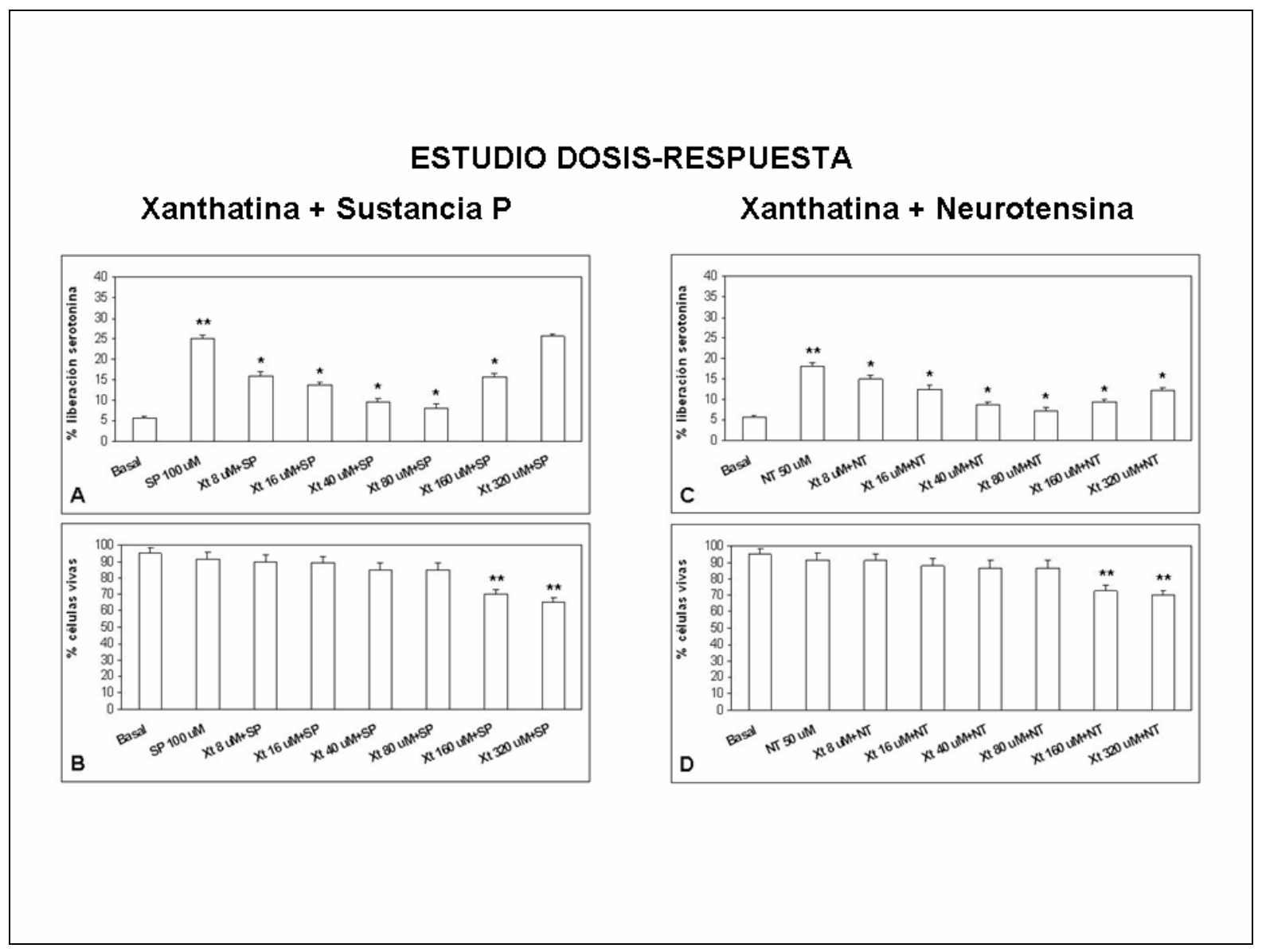

Figura 2. A: Efecto de concentraciones crecientes de xanthatina $(\mathrm{Xt})$ sobre la liberación de serotonina mastocitaria inducida por sustancia P $100 \mu \mathrm{M}(\mathrm{SP})$. B: Efecto de concentraciones crecientes de xanthatina (Xt) más sustancia P 100 $\mu \mathrm{M}(\mathrm{SP})$ sobre la vitalidad mastocitaria. C: Efecto de concentraciones crecientes de xanthatina (Xt) sobre la liberación de serotonina mastocitaria inducida por neurotensina $50 \mu \mathrm{M}$ (NT). D: Efecto de concentraciones crecientes de xanthatina $(\mathrm{Xt})$ más neurotensina $50 \mu \mathrm{M}(\mathrm{NT})$ sobre la vitalidad mastocitaria. Los resultados se expresaron como porcentaje de liberación de serotonina $(A$ y $C$ ) o como porcentaje de células vivas ( $B$ y $D$ ). Los valores se presentan como media \pm S.E.M. ${ }^{*} \mathrm{P}<0,01$ versus basal $y{ }^{*} \mathrm{P}<0,01$ versus sustancia $\mathrm{P}$.

dosis de xanthatina de $16 \mu \mathrm{M}$ para llevar a cabo los estudios morfológicos.

La incubación de mastocitos con xanthatina (dosis de $8 \mu \mathrm{M}, 16 \mu \mathrm{M}, 40 \mu \mathrm{M}$ y 80 ) inhibió $(P<0,01)$, en forma dosis-dependiente, la liberación de serotonina inducida por neurotensina 50 $\mu \mathrm{M}$ (Fig. 2C), sin modificar la viabilidad celular (Fig. 2D). La incubación de las células con xanthatina $160 \mu \mathrm{M}$ y $320 \mu \mathrm{M}$ aumentó la liberación de serotonina con respecto a la dosis $\begin{array}{llllll}\text { de } & 80 & \mu \mathrm{M} & \text { (Fig. } & 2 \mathrm{C}) & \text { y disminuyó }\end{array}$ significativamente $(P<0,01)$ la vitalidad celular (Fig. 2D). En base a estos resultados, se seleccionó la dosis de xanthatina de $16 \mu \mathrm{M}$ para llevar a cabo los estudios morfológicos.

Estudios morfológicos

Microscopía óptica
Los mastocitos se identificaron por la presencia en el citoplasma de gránulos secretorios metacromáticos. Los mastocitos del grupo basal presentaron alta densidad granular (Figura 3A). Los mastocitos estimulados con sustancia P 100 $\mu \mathrm{M}$ (Fig. 3B) y con neurotensina $50 \mu \mathrm{M}$ (Fig. 3C) mostraron un evidente proceso de degranulación. Los mastocitos pretratados con xanthatina $16 \mu \mathrm{M}$ y luego estimulados con sustancia $\mathrm{P} 100$ $\mu \mathrm{M}$ (Fig. 3D) o con neurotensina $50 \mu \mathrm{M}$ (Fig. 3E) presentaron morfología similar a la del grupo basal.

Microscopía electrónica de transmisión

Los mastocitos basales mostraron microvellosidades en la superficie celular y numerosos gránulos de secreción, electrónicamente densos, regularmente distribuidos en todo el citoplasma. (Fig. 3F). Los mastocitos estimulados con 


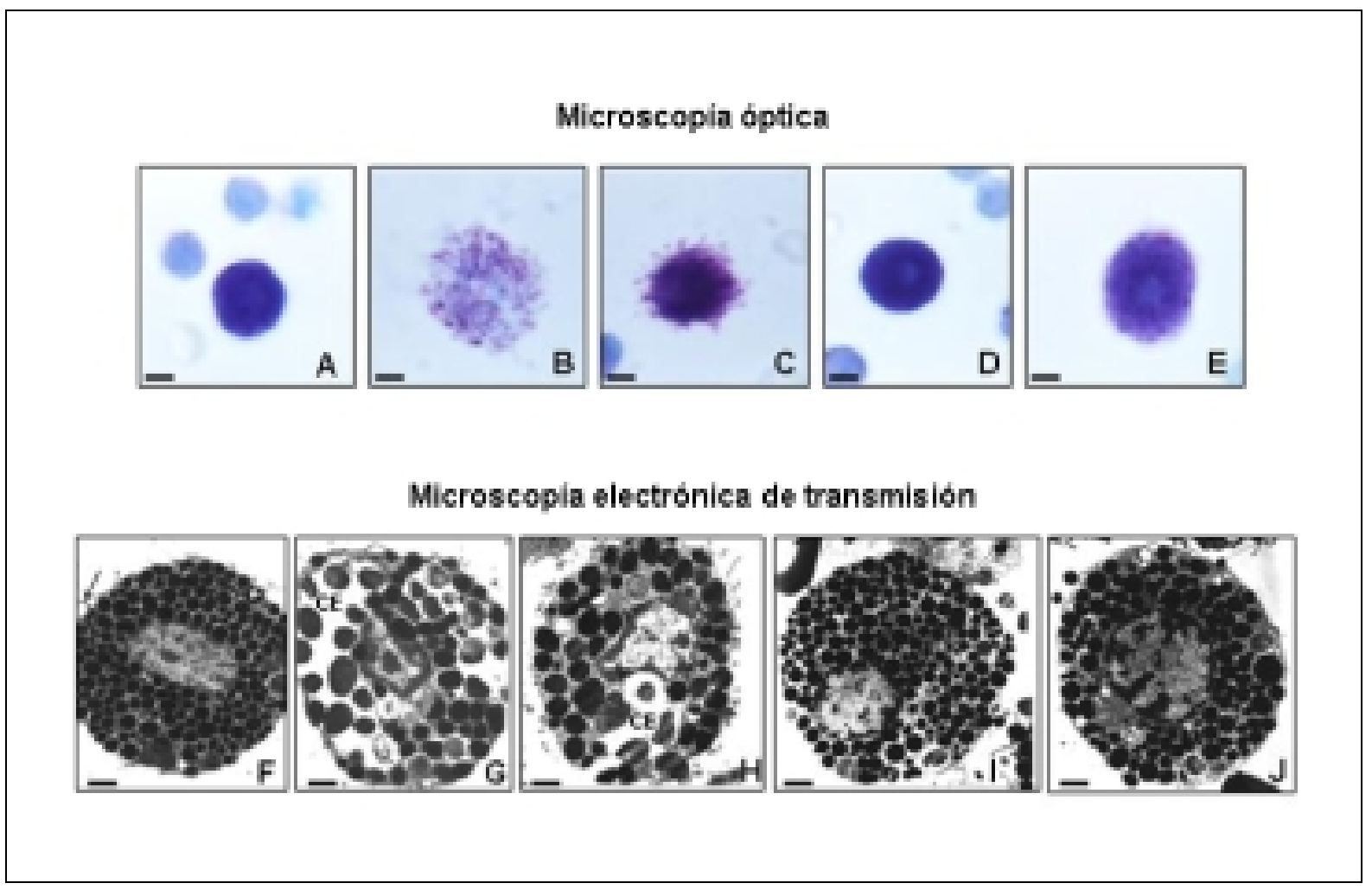

Figura 3. A, B, C, D y E: Microscopía óptica de mastocitos peritoneales de rata coloreados con azul de toluidina. A: Basal. B: Sustancia P $100 \mu$ M. C: Neurotensina $50 \mu$ M. D: Xanthatina $16 \mu$ M + Sustancia P $100 \mu$ M. E: Xanthatina 16 $\mu \mathrm{M}+$ Neurotensina $50 \mu \mathrm{M}$. Barra $=10 \mu \mathrm{m}$. F, G, H, I y J: Microscopía electrónica de transmisión de mastocitos peritoneales de rata. A: Basal. B: Sustancia P $100 \mu \mathrm{M}$. C: Neurotensina $50 \mu \mathrm{M}$. D: Xanthatina $16 \mu \mathrm{M}+$ Sustancia P 100 $\mu \mathrm{M}$. E: Xanthatina $16 \mu \mathrm{M}+$ Neurotensina $50 \mu \mathrm{M}$. Barra $=3 \mu \mathrm{m}$. Flecha negra: microvellosidad. Flecha blanca: gránulo citoplasmático homogéneamente denso. CE: canal de exocitosis.

sustancia P $100 \mu \mathrm{M}$ (Fig. 3G) y con neurotensina $50 \mu \mathrm{M}(\mathrm{Fig} 3 \mathrm{H})$ mostraron gránulos secretorios irregulares con diferentes grados de densidad electrónica y canales de exocitosis. Los mastocitos pretratados con xanthatina $16 \mu \mathrm{M}$ y luego estimulados con sustancia P $100 \mu \mathrm{M}$ (Fig. 3I) o con neurotensina $50 \mu \mathrm{M}$ (Fig. 3J) mostraron mínima degranulación y características morfológicas similares al grupo basal.

Estudios de estabilidad química de xanthatina El valor de Rf para xanthatina fue de 0,46 . No se observaron cambios de $\mathrm{Rf}$ ni bandas de degradación de xanthatina luego de las incubaciones.

\section{DISCUSIÓN}

Los primeros experimentos del presente trabajo fueron diseñados para evaluar el efecto de xantatina sobre la respuesta secretoria inducida por sustancia $P$ y neurotensina en mastocitos peritoneales. Esta respuesta secretoria fue medida a través de la liberación de serotonina, mediador preformado asociado a la matriz de los gránulos citoplasmáticos. La serotonina es utilizada como un marcador bioquímico de activación de mastocitos, de acuerdo con los trabajos de Taylor et al (1995), Kim et al (1997) y Kawamoto et al (2002).

La sustancia $P$ y la neurotensina son secretagogos de mastocitos capaces de estimular la liberación de serotonina y otros mediadores de la inflamación a partir de los gránulos intracitoplasmáticos (Kulka et al, 2008; Katsanos et al, 2008). La sustancia P ejerce su acción por activación de receptores NK1 acoplados a proteínas $\mathrm{G}$ heterotriméricas. La estimulación de estos receptores conduce a la activación de fosfolipasa $\mathrm{C}$, con la consiguiente producción de inositol trifosfato y diacilglicerol, que conducen al aumento intracelular de calcio (Schäffer et al, 1998). La neurotensina estimula receptores específicos NTR, acoplados también 
a proteínas $\mathrm{G}$ heterotriméricas (Castagliuolo et al., 1999).

Los primeros resultados de nuestro estudio demostraron que la incubación de mastocitos peritoneales con soluciones de sustancia $P 100$ $\mu \mathrm{M}$ y neurotensina $50 \mu \mathrm{M}$ incrementa la liberación de serotonina con respecto al grupo basal y que este efecto es inhibido por xanthatina en forma dosis-dependiente. Los resultados obtenidos a partir de los ensayos con azul tripán demostraron que xantahtina no resultó citotóxica, excepto a concentraciones mayores de $160 \mu \mathrm{M}$.

A pesar de las fuertes evidencias bioquímicas, consideramos que se necesitaba además una evaluación morfológica para reforzar la validez de nuestros hallazgos iniciales. Así, diseñamos un segundo conjunto de experimentos para analizar el efecto de la xanthatina sobre la morfología mastocitaria, a nivel de microscopía óptica y electrónica.

Nuestros hallazgos morfológicos, a nivel de la microscopía óptica, demostraron que la xanthatina inhibió la liberación de gránulos metacromáticos mastocitarios, sugiriendo una interacción del sesquiterpeno con la población de mastocitos y una inhibición de la degranulación inducida por la sustancia $\mathrm{P}$ y la neurotensina.

Además, en nuestro estudio, la liberación significativamente aumentada de serotonina después del tratamiento con sustancia $P$ y con neurotensina, se relacionó estrechamente con evidentes cambios ultraestructurales mastocitarios. A pesar de que xanthatina indujo algunos cambios a este nivel, su acción fue significativamente menos dramática que la ejercida por los secretagogos "per se". Algunos autores han demostrado que, a nivel de la microscopía electrónica de transmisión, los gránulos de los mastocitos basales son homogéneamente densos (Lawson et al, 1977; Dvorak, 2005; Pickett y Edwardson, 2006). Se ha descripto, además, que la liberación de mediadores mastocitarios está asociada con alteraciones fácilmente detectables en la apariencia ultraestructural de los gránulos, que incluyen un agrandamiento del espacio entre el gránulo y su membrana, edematización granular, disminución de la densidad electrónica del contenido granular y presencia de canales de exocitosis.

El hecho que los mastocitos tratados con xanthatina antes de la sustancia $P$ o de la neurotensina no mostraran evidencias de degranulación podría indicar: 1) cambios en la capacidad de los mastocitos para responder completamente a los secretagogos o: 2) inactivación del secretagogo por interacción química con el sesquiterpeno. Esta segúnda posibilidad implicaría cambios estructurales en xanthatina que podrían ser detectadas por cromatografía en capa fina. Los valores de Rf obtenidos a partir de los estudios de estabilidad por cromatografía en capa fina nos permitieron concluir que xanthatina se mantuvo estable durante los experimentos y que, además, no existe unión entre el sesquiterpeno y los secretagogos.

A pesar de que no es posible establecer una correlación estricta entre estudios "in vitro" e "in vivo", el hecho de que xanthatina inhiba la liberación de serotonina a partir de mastocitos peritoneales, plantea la posibilidad de que el sesquiterpeno podría actuar como un estabilizador mastocitario en el animal intacto.

En conclusión, los resultados obtenidos en el presente trabajo permiten concluir que xanthatina inhibe la degranulación de mastocitos inducida por sustancia $\mathrm{P}$ y por neurotensina. Estudios adicionales acerca de los mecanismos de los mecanismos de acción del sequiterpeno permitirán un mejor conocimiento de los cambios celulares y moleculares que tienen lugar en los mastocitos después del tratamiento con xanthatina. Los presentes hallazgos fortalecen la posibilidad de que este sesquiterpeno de origen natural pueda representar una nueva alternativa en el diseño de agentes farmacológicos y en el tratamiento de patologías vinculadas con la activación de mastocitos.

\section{BIBLIOGRAFÍA}

Blank U, Rivera J. 2004. The ins and outs of IgEdependent mast cell exocytosis. Trends Immunol 25:266-273.

Calderón GM, Torres-López J, Lin T, Chavez B, Hernández M, Muñoz O, Befus AD, Enciso JA. 1998. Effects of toxin A from Clostridium difficile on mast cell activation and survival. Infect Immun 66:2755-2761.

Castagliuolo I, Wang CC, Valenick L, Pasha A, Nikulasson S, Carraway RE, Pothoulakis C. 1999. Neurotensin is a proinflammatory neuropeptide in colonic inflammation. J Clin Invest 103:843-849.

Dvorak AM. 2005. Ultrastructural studies of human basophils and mast cells. J Histochem Cytochem 53:1043-1070.

Favier LS, Maria AOM, Wendel GH, Borkowski EJ, Giordano OS, Pelzer L, Tonn CE. 2005. Anti-ulcerogenic activity of xanthanolide sesquiterpenes from Xanthium cavanillesii in rats. J Ethnopharmacol 100:260-267. 
Galli SJ, Grimbaldeston M, Tsai M. 2008. Immunomodulatory mast cells: negative, as well as positive, regulators of immunity. Nature 8:478-486.

Giordano OS, Guerreiro E, Pestchanker MJ. 1990. The gastric cytoprotective effect of several sesquiterpene lactones. J Nat Prod 53:803-809.

Gurish MF, Austen KF. 2001. The diverse roles of mast cells. J Exp Med 194:F1-F5.

Kalesnikoff J, Galli SJ. 2008. New developments in mast cell biology. Nature Immunology 9:1215-1223.

Katsanos GS, Anogianaki A, Castellani ML, Ciampoli C, De Amicis D, Orso C, Pollice $R$, Vecchiet J, Tetè $S$, Salini $V$, Caraffa $A$, Patruno A, Shaik YB, Kempuraj D, Doyle $R$, Antinolfi PL, Cerulli G, Conti CM, Fulcheri M, Neri G, Sabatino G. 2008. Biology of neurotensin: revisited study. Int $J$ Immunopathol Pharmacol 21:255-259.

Kawamoto K, Aoki J, Tanaka A, Itakura A, Hosono H, Arai H, Kiso Y, Matsuda H. 2002. Nerve growth factor activates mast cells through the collaborative interaction with lysophosphatidylserine expressed on the membrane surface of activated platelets. J Immunol 168:6412-6419.

Kim TD, Eddlestone GT, Mahmoud SF, Kuchtey J, Fewtrell C. 1997. Correlating Ca2+ responses and secretion in individual $\mathrm{RBL}$ 2H3 mucosal mast cells. J Biol Chem 272:31225-31229.

Kubes $P$, Granger DN. 1996. Leukocyteendothelial cell interactions evoked by mast cells. Cardiovasc Res 32:699-708.

Kulka M, Sheen CH, Tancowny BP, Grammer LC, Schleimer RP. 2008. Neuropeptides activate human mast cell degranulation and chemokine production. Immunology 123:398410.

Kushnir-Sukhov NM, Brown JM, Wu Y, Kirshenbaum A, Metcalfe DD. 2007. Human mast cells are capable of serotonin synthesis and release. J Allergy Clin Immunol 119:498499.

Lawson D, Raff MC, Gomperts B, Fewtrell C, Gilula NB. 1977. Molecular events during membrane fusion. A study of exocytosis in rat peritoneal mast cells. J Cell Biol 72:242-259.

Moriyama $M$, Sato $T$, Inoue $H$, Fukuyama $S$, Teranishi $H$, Kangawa $K$, Kano $T$, Yoshimura A, Kojima M. 2005. The neuropeptide neuromedin $U$ promotes inflammation by direct activation of mast cells. J Exp Med 202:21724.

Penissi A, Rudolph I, Fogal T, Piezzi R. 2003a. Changes in duodenal mast cells in response to dehydroleucodine. Cells Tissues Organs
173:234-241.

Penissi AB, Fogal T, Guzmán JA, Piezzi RS. 1998. Gastroduodenal mucosal protection induced by dehydroleucodine. Mucus secretion and role of monoamines. Dig Dis Sci 43:791-798.

Penissi AB, Giordano OS, Guzmán JA, Rudolph MI, Piezzi RS. 2006. Chemical and pharmacological properties of dehydroleucodine, a lactone isolated from Artemisia douglasiana Besser. Molecular Medicinal Chemistry 10:1-11.

Penissi AB, Mariani ML, Souto M, Guzmán JA, Piezzi RS. 2000. Changes on gastroduodenal 5hydroxytryptamine-containing cells induced by dehydroleucodine. Cells, Tissues and Organs 166:259-266.

Penissi AB, Piezzi RS. 1999. Effect of dehydroleucodine on mucus production. A quantitative study. Dig Dis Sci 44:708-712.

Penissi AB, Rudolph MI, Piezzi RS. 2003b. Gastrointestinal mucosal protection induced by dehydroleucodine: role of mast cells (review). Biocell 27:163-172.

Penissi AB, Rudolph MI, Villar M, Coll RC, Fogal TH, Piezzi RS. 2003c. Effect of dehydroleucodine on histamine and serotonin release from mast cells in the isolated mouse jejunum. Inflamm Res 52:199-205.

Penissi AB, Vera ME, Mariani ML, Rudolph MI, Ceñal JP, de Rosas JC, Fogal TH, Tonn CE, Favier LS, Giordano OS, Piezzi RS. 2009. Novel anti-ulcer alpha,beta-unsaturated lactones inhibit compound 48/80-induced mast cell degranulation. Eur J Pharmacol 612:122130.

Pickett JA, Edwardson JM. 2006. Compound exocytosis: mechanisms and functional significance. Traffic 7:109-116.

Rao KN, Brown MA. 2008. Mast cells: multifaceted immune cells with diverse roles in health and disease. Ann N Y Acad Sci 1143:83-104.

Richardson JD, Vasko MR. 2002. Cellular mechanisms of neurogenic inflammation. J Pharmacol Exp Ther 302:839-845.

Schäffer M, Beiter T, Becker HD, Hunt TK. 1998. Neuropeptides: mediators of inflammation and tissue repair? Arch Surg. 133:1107-1116,

Schmelz M, Petersen LJ. 2001. Neurogenic inflammation in human and rodent skin. News Physiol Sci 16:33-37.

Taylor AM, Galli SJ, Coleman JW. 1995. Stemcell factor, the kit ligand, induces direct degranulation of rat peritoneal mast cells in vitro and in vivo: dependence of the in vitro effect on period of culture and comparisons of 
stem-cell factor with other mast cell-activating agents. Immunology 86: 427-433.

Wagner J, Vitali P, Palfreyman MG, Zraika $M$ and Huot S. 1982. Simultaneous determination of 3,4-dihydroxyphenylalanine, 5-hydroxytryptophan, dopamine, 4-hydroxy-3methoxyphenylalanine, norepinephrine, 3,4- dihydroxyphenylacetic acid, homovanillic acid, 5-hydroxytryptamine, and 5-hydroxyindoleacetic acid in rat cerebrospinal fluid and brain by high performance liquid chromatography with electrochemical detection. J Neurochem 38:1241-1254. 